\title{
VISCOSITY SOLUTIONS OF FULLY NONLINEAR FUNCTIONAL PARABOLIC PDE
}

\author{
LIU WEI-AN AND LU GANG
}

Received 17 October 2004 and in revised form 21 October 2005

By the technique of coupled solutions, the notion of viscosity solutions is extended to fully nonlinear retarded parabolic equations. Such equations involve many models arising from optimal control theory, economy and finance, biology, and so forth. The comparison principle is shown. Then the existence and uniqueness are established by the fixed point theory.

\section{Introduction}

In this paper, we are concerned with the following problem:

$$
\begin{gathered}
f\left(t, x ; u, u_{t}(\tau) ; \partial_{t} u, D_{x} u, D_{x}^{2} u\right)=0, \quad(t, x) \in Q, \\
u(t, x)=0, \quad(t, x) \in \Gamma, \\
u(t, x)=\phi(t, x), \quad(t, x) \in Q_{\bar{\tau}} .
\end{gathered}
$$

Here, $Q=(0, T) \times \Omega, \Gamma=(0, T) \times \partial \Omega(T<+\infty$ or $T=+\infty)$, and $\Omega$ is a bounded open subset of $\mathbb{R}^{n}, Q_{\bar{\tau}}=[-\bar{\tau}, 0] \times \Omega, C^{m}=C[-\bar{\tau}, 0] \times \cdots \times C[-\bar{\tau}, 0]$ denotes the corresponding continuous function space; $u: Q_{\bar{\tau}} \cup Q \rightarrow \mathbb{R}$ is the unknown function, $u_{t}(\tau)=\left(u_{t}\left(\tau_{1}\right), \ldots\right.$, $\left.u_{t}\left(\tau_{m}\right)\right)=\left(u\left(t+\tau_{1}, x\right), \ldots, u\left(t+\tau_{m}, x\right)\right)$ with $-\bar{\tau} \leq \tau_{j} \leq 0, j=1,2, \ldots, m, f: Q \times \mathbb{R} \times C^{m} \times$ $\mathbb{R} \times \mathbb{R}^{n} \times \mathbf{S}(n) \rightarrow \mathbb{R}$ is a given functional which is locally bounded, $\mathbf{S}(n)$ denotes the set of real symmetric $n \times n$ matrices equipped with its usual order, that is, $X \leq Y$ implies that $\xi^{t} X \xi \leq \xi^{t} Y \xi$ for every $\xi \in \mathbb{R}^{n} ; D_{x} u$ and $D_{x}^{2} u$ denote the gradient and Hessian matrix of the function $u$ with respect to the argument $x ; \phi(t, x) \in C\left(\bar{Q}_{\bar{\tau}}\right)$ and $\left.\phi(t, x)\right|_{\Gamma}=0$.

The functional $f$ is said to be degenerate parabolic if

$$
f\left(t, x ; u, u_{t}(\tau) ; a, p, X\right)-\left(t, x ; u, u_{t}(\tau) ; a, p, Y\right) \geq 0
$$

for all $\left(t, x ; u, u_{t}(\tau) ; a, p\right) \in Q \times \mathbb{R} \times C^{m} \times \mathbb{R} \times \mathbb{R}^{n}, X \leq Y \in \mathbf{S}(n)$,

$$
f\left(t, x ; u, u_{t}(\tau) ; a, p, X\right)-f\left(t, x ; u, u_{t}(\tau) ; b, p, X\right) \geq 0
$$

for all $\left(t, x ; u, u_{t}(\tau) ; p, X\right) \in Q \times \mathbb{R} \times C^{m} \times \mathbb{R}^{n} \times \mathbf{S}(n), a \geq b \in \mathbb{R}$. 
Clearly, linear or semilinear parabolic equations satisfy the inequalities (1.2) and (1.3).

The problem (1.1) involves many examples including the initial problems for ordinary retarded differential equation of the first order, the initial-boundary value problems for linear and quasilinear parabolic and degenerate parabolic retarded partial differential equations (which model the reaction-diffusion phenomena in chemistry and biology), and fully nonlinear functional partial differential equations (such as MongeAmpere equation in geometry, and Bellman and Isaacs equations arising from optimal control).

In addition, to provide a novel concept of weak solution to fully nonlinear partial differential equations, the method of viscosity solution can be used to prove the convergence of difference scheme for some fully nonlinear problems (see [4]).

As it is well known, the theory of viscosity solution is based on the comparison. Unfortunately, such a principle for the functional equation is not necessarily true (see Remark 2.3). However, we extended the method of viscosity solution to monotonic functional parabolic PDE by applying the technique of coupled solutions (see $[5,6,7])$. In this paper, we introduce the notion of coupled viscosity sub- and super-solutions in the strong sense, similar to that in $[2,3]$, to overcome the lack of monotonicity such that the method of viscosity solution can be applied to nonmonotonic functional parabolic PDE.

We organize our paper as follows. In Section 2, we present the basic concepts and main result. We discuss the comparison principle in Section 3. In Section 4, we prove the existence and uniqueness theorem. In Section 5, we construct a coupled viscosity sub- and super-solution in the strong sense to (1.1) by making use of its conventional viscosity suband super-solution and the solution of a related retarded ordinary differential inequality. In Section 6, the result is applied to retarded Bellman equation.

\section{Coupled viscosity sub- and super-solutions in the strong sense}

The sets $\mathscr{P}_{\Omega}^{2,+} u(t, x)$ (or $\mathscr{P}_{\Omega}^{2,-} u(t, x)$ ) and $\overline{\mathscr{P}}_{\Omega}^{2,+} u(t, x)$ (or $\overline{\mathscr{P}}_{\Omega}^{2,-} u(t, x)$ ) denote, respectively, the parabolic superjet (or subjet) of the second order for the function $u$ at $(t, x)$ and its closure defined as that in [1], that is,

$$
\begin{aligned}
\mathscr{P}_{\Omega}^{2,+} u(t, x)=\{ & (a, p, X) \in \mathbb{R} \times \mathbb{R}^{n} \times \mathbf{S}(n): u(s, y) \leq u(t, x)+a(s-t)+\langle p, y-x\rangle \\
& \left.+\frac{1}{2}\langle X(y-x), y-x\rangle+o\left(|s-t|+|y-x|^{2}\right), \text { as }(s, y) \longrightarrow(t, x),(s, y) \in \mathcal{2}\right\}, \\
\mathscr{P}_{\Omega}^{2,-} u(t, x)= & (b, q, Y) \in \mathbb{R} \times \mathbb{R}^{n} \times \mathbf{S}(n): u(s, y) \geq u(t, x)+b(s-t)+\langle q, y-x\rangle \\
& \left.+\frac{1}{2}\langle Y(y-x), y-x\rangle+o\left(|s-t|+|y-x|^{2}\right), \text { as }(s, y) \longrightarrow(t, x),(s, y) \in \mathscr{2}\right\}, \\
\mathscr{P}_{\Omega}^{2,+} u(t, x)=\{ & (a, p, X) \in \mathbb{R} \times \mathbb{R}^{n} \times \mathbf{S}(n): \exists\left(t_{k}, x_{k}\right) \in \mathscr{2}, \\
& \left(a_{k}, p_{k}, X_{k}\right) \in \mathscr{P}_{\Omega}^{2,+} u\left(t_{k}, x_{k}\right), \text { s.t., as } \longrightarrow \infty, \\
& \left.\left(t_{k}, x_{k}, u\left(t_{k}, x_{k}\right), a_{k}, p_{k}, X_{k}\right) \longrightarrow(t, x, u(t, x), a, p, X)\right\},
\end{aligned}
$$




$$
\begin{aligned}
\overline{\mathscr{P}}_{\Omega}^{2,-} u(t, x)=\{ & (b, q, Y) \in \mathbb{R} \times \mathbb{R}^{n} \times \mathbf{S}(n): \exists\left(t_{k}, x_{k}\right) \in \mathscr{2}, \\
& \left(b_{k}, q_{k}, Y_{k}\right) \in \mathscr{P}_{\Omega}^{2,-} u\left(t_{k}, x_{k}\right), \text { s.t., as } k \longrightarrow \infty, \\
& \left.\left(t_{k}, x_{k}, u\left(t_{k}, x_{k}\right), b_{k}, q_{k}, Y_{k}\right) \longrightarrow(t, x, u(t, x), b, q, Y)\right\} .
\end{aligned}
$$

To extend the technique of viscosity solutions to equations without smoothness which arise from optimal control and economic problems, for example, random parabolic equations or systems, we will use the following notations:

$$
\begin{aligned}
& u^{*}(t, x)=\lim _{r \rightarrow 0} \sup \{u(s, y)|| t-s|+| x-y \mid<r\}, \\
& u_{*}(t, x)=\liminf _{r \rightarrow 0}\{u(s, y)|| t-s|+| x-y \mid<r\},
\end{aligned}
$$

which are called the upper and lower semicontinuous envelopes of $u$, respectively. $f^{*}(t$, $\left.x ; u, u_{t}(\tau), a, p, X\right)$ and $f_{*}\left(t, x ; u, u_{t}(\tau), a, p, X\right)$ are defined similarly.

We first give the conventional definition for viscosity sub- and super-solutions.

Definition 2.1. Suppose that $f$ is degenerate parabolic and locally bounded. $U(t, x)(V(t$, $x)$ ) is called a viscosity subsolution (super-solution) of (1.1) if it is locally bounded in $Q_{\bar{\tau}} \cup$ $Q$ such that

$$
\begin{gathered}
f_{*}\left(t, x ; U^{*}, U_{t}(\tau)^{*} ; a, p, X\right) \leq 0, \quad \forall(t, x) \in Q,(a, p, X) \in \mathscr{P}_{\Omega}^{2,+} U^{*}(t, x), \\
\left(f^{*}\left(t, x ; V_{*}, V_{t *}(\tau) ; b, q, Y\right) \geq 0, \quad \forall(t, x) \in Q,(b, q, Y) \in \mathscr{P}_{\Omega}^{2,-} V_{*}(t, x)\right), \\
U^{*}(t, x) \leq 0, \quad \text { for }(t, x) \in \Gamma, \quad\left(V_{*}(t, x) \geq 0, \text { for }(t, x) \in \Gamma\right), \\
U^{*}(t, x) \leq \phi(t, x), \text { for }(t, x) \in Q_{\bar{\tau}}, \quad\left(V_{*}(t, x) \geq \phi(t, x), \text { for }(t, x) \in Q_{\bar{\tau}}\right) .
\end{gathered}
$$

Definition 2.2. $u(t, x)$ is called a viscosity solution of $(1.1)$ if it is both a viscosity subsolution and a viscosity super-solution of (1.1).

Remark 2.3. For such defined viscosity sub- and super-solutions, the comparison principle does not hold in general. For example, consider

$$
\begin{gathered}
\frac{\partial u}{\partial t}-\frac{\partial^{2} u}{\partial x^{2}}-u+u_{t}\left(\frac{\pi}{2}\right)=0, \quad t>0,0<x<\pi \\
u(t, 0)=u(t, \pi)=0, \quad t>0 \\
u(t, x)=\sin x \cos t, \quad-\frac{\pi}{2}<t<0,0<x<\pi
\end{gathered}
$$

We observe that

$$
V(t, x)=\sin x \cos t, \quad U(t, x)=0
$$

are a viscosity super-solution and a viscosity subsolution, respectively, but

$$
V(t, x) \geq U(t, x)
$$

does not hold everywhere. 
3542 Viscosity solutions to functional parabolic PDE

For that reason, we introduce the following notion of the coupled viscosity sub- and super-solution in the strong sense similar to that in [3] of Ishii and Koike and [2] of Engler and Lenhart, and then the comparison principle will hold.

Definition 2.4. Suppose that $(U(t, x), V(t, x))$ satisfies the following condition:

(C) there is a function $\psi(t, x) \in C\left(Q_{\bar{\tau}} \cup Q\right)$ between $U$ and $V$, that is,

$$
U^{*} \leq \psi \leq V_{*} \quad \text { or } \quad V_{*} \leq \psi \leq U^{*} .
$$

$(U, V)$ is called a coupled viscosity sub-and super-solution in the strong sense of (1.1) if it is locally bounded in $Q_{\bar{\tau}} \cup Q$ and satisfies that

$$
\begin{gathered}
f_{*}\left(t, x ; U^{*}, \psi_{t}(\tau) ; a, p, X\right) \leq 0 \leq f^{*}\left(t, x ; V_{*}, \psi_{t}(\tau) ; b, q, Y\right), \\
\forall(t, x) \in Q, \\
(a, p, X) \in \mathscr{P}_{\Omega}^{2,+} U^{*}(t, x), \quad(b, q, Y) \in \mathscr{P}_{\Omega}^{2,-} V_{*}(t, x),
\end{gathered}
$$

and (2.4) for any continuous function $\psi(t, x)$ between $U$ and $V$.

To establish our main results, the following conditions are needed.

(H1) There exists a real number $\beta>0$ such that

$$
f_{*}(t, x ; r, W ; a, p, X)-f^{*}(t, x ; r, W ; b, p, X) \geq \beta(a-b),
$$

for $a \geq b,(t, x ; r, W ; p, X) \in \bar{Q} \times \mathbb{R} \times \mathbb{R}^{m} \times \mathbb{R}^{n} \times \mathbf{S}(n)$.

$(\mathrm{H} 2)$ There is a continuous function $\omega:(0,+\infty) \rightarrow(0,+\infty)$ that satisfies $\omega\left(0^{+}\right)=0$ such that for each fixed $t \in \mathbb{R}^{+}$,

$$
f^{*}(t, y ; r, W ; a, \alpha(x-y), Y)-f_{*}(t, x ; r, W ; a, \alpha(x-y), X) \leq \omega\left(\alpha|x-y|^{2}+|x-y|\right),
$$

whenever $t \in(0, T), x, y \in \Omega, r, a, \alpha \in \mathbb{R}, W \in \mathbb{R}^{m}$, and $X, Y \in \mathbf{S}(n)$ satisfying

$$
-3 \alpha\left(\begin{array}{cc}
I & 0 \\
0 & I
\end{array}\right) \leq\left(\begin{array}{cc}
X & 0 \\
0 & -Y
\end{array}\right) \leq 3 \alpha\left(\begin{array}{cc}
I & -I \\
-I & I
\end{array}\right) .
$$

(H3) There exists a real number $\gamma>0$ such that

$$
f_{*}(t, x ; r, W ; a, p, X)-f^{*}(t, x ; s, W ; a, p, X) \geq-\gamma(r-s),
$$

for $r \geq s,(t, x ; W ; a, p, X) \in \bar{Q} \times \mathbb{R}^{m} \times \mathbb{R} \times \mathbb{R}^{n} \times \mathbf{S}(n)$. 
(H4) There are real numbers $L_{k}>0, k=1, \ldots, m$, such that

$$
\begin{gathered}
f_{*}\left(t, x ; r, U_{t}(\tau) ; a, p, X\right)-f^{*}\left(t, x ; r, V_{t}(\tau) ; a, p, X\right) \\
\leq \sum_{k=1}^{m} L_{k}\left|U\left(t+\tau_{k}, x\right)-V\left(t+\tau_{k}, x\right)\right|,
\end{gathered}
$$

for $(t, x ; r ; a, p, X) \in \bar{Q} \times \mathbb{R} \times \mathbb{R} \times \mathbb{R}^{n} \times \mathbf{S}(n)$.

Remark 2.5. (H2) and (H3) are basic conditions in the theory of viscosity solutions. The equation is called forward parabolic if (H1) holds and is proper under the conditions (H1) and (H3). The Lipschitz condition (H4) is applied to construct a contraction mapping.

\section{The comparison}

We first show the comparison principle.

Theorem 3.1. Let $f$ be degenerate parabolic and locally bounded. Suppose that the conditions (H1)-(H4) hold. If $\{U, V\}$ is a coupled viscosity sub-and super-solution in the strong sense of (1.1), then

$$
U^{*} \leq V_{*}, \quad \text { in } \bar{Q}_{\bar{\tau}} \cup \bar{Q} \text {. }
$$

Proof. For any given continuous function $\psi(t, x)$ between $U$ and $V$,

$$
\begin{gathered}
f\left(t, x ; u, \psi_{t}(\tau) ; \partial_{t} u, D_{x} u, D_{x}^{2} u\right)=0, \quad(t, x) \in Q, \\
u(t, x)=0, \quad(t, x) \in \Gamma, \\
u(t, x)=\phi(t, x), \quad(t, x) \in Q_{\bar{\tau}},
\end{gathered}
$$

is an initial-boundary value problem of second-order fully nonlinear PDE without delay. It can be easily shown that $U$ and $V$ are also viscosity sub- and super-solutions, to (3.2), respectively.

By (H4) and the continuity of $\psi$, condition (H2) holds for the problem (3.2). Similar to the proof of [1, Theorem 8.2] (where the equation reads $\partial_{t} u+f\left(t, x ; u, D_{x} u, D_{x}^{2} u\right)=0$ ), it can be shown that the comparison principle for the problem (3.2) holds, too.

The above derivation is independent of $T$ which is an arbitrary finite number, hence the comparison principle holds for all the positive $t$. The proof is completed.

\section{The existence and uniqueness}

Now, we prove existence and uniqueness by making use of the fixed point theorem.

Theorem 4.1. Let $f$ be degenerate parabolic, locally bounded, and satisfy (H1)-(H4). Suppose that $(U, V)$ is a coupled viscosity sub-and super-solution in the strong sense of (1.1) with $U^{*}(t, x)=V_{*}(t, x)=0$, for $(t, x) \in \Gamma$ and $U^{*}>-\infty, V_{*}<+\infty$. Then, there is a unique viscosity solution $u(t, x) \in C\left(\bar{Q}_{\bar{\tau}} \cup \bar{Q}\right)$ satisfying

$$
U^{*} \leq u \leq V_{*}, \quad \text { on } \bar{Q}_{\bar{\tau}} \cup \bar{Q} .
$$


3544 Viscosity solutions to functional parabolic PDE

Proof. We assume first that $T<+\infty$.

To prove the theorem, we construct first an operator $A$ by conventional existence of viscosity solution, then we show that $A$ is a contraction mapping, thus by Banach fixed point theorem, we know that $A$ has a unique fixed point which is just the viscosity solution to (1.1).

To construct an operator $A$, let us consider the problem (3.2) for an arbitrarily given function $\psi$ between $U$ and $V$.

It is clear that $U$ and $V$, a coupled viscosity sub- and super-solution in the strong sense for (1.1), are a pair of general viscosity sub- and super-solutions of (3.2). Thanks to $(\mathrm{H} 1)-(\mathrm{H} 4)$, similar to [1, Theorems 8.1 and 8.2 ], we can prove that the comparison principle for (3.2) holds and there is a unique viscosity $\tilde{u}(t, x) \in C(Q)$, which depends on $\psi$ naturally, and satisfies $U^{*} \leq \tilde{u} \leq V_{*}$. We define that

$$
u(t, x)= \begin{cases}\phi(t, x), & (t, x) \in[-\bar{\tau}, 0] \times \Omega, \\ \tilde{u}(t, x), & (t, x) \in Q \times \Omega .\end{cases}
$$

Then we have an operator $A: C\left(\bar{Q}_{\bar{\tau}} \cup \bar{Q}\right) \rightarrow C\left(\bar{Q}_{\bar{\tau}} \cup \bar{Q}\right)$ defined by

$$
u=A \psi
$$

Clearly, the fixed point of $A$ in $C\left(\bar{Q}_{\bar{\tau}} \cup \bar{Q}\right)$ is a viscosity solution of (1.1).

Secondly, we show that the operator $A$ is a contraction mapping in a subset of $C\left(\bar{Q}_{\bar{\tau}} \cup\right.$ Q).

Prior to doing so, instead of (H3), we might suppose as well that, $\left(\mathrm{H} 3^{*}\right)$ for a large enough $\lambda>0$,

$$
f_{*}(t, x ; r, W ; a, p, X)-f^{*}(t, x ; s, W ; a, p, X) \geq \lambda(r-s)
$$

whenever $r \geq s \in \mathbb{R},(t, x ; W ; a, p, X) \in \bar{Q} \times \mathbb{R}^{m} \times \mathbb{R} \times \mathbb{R}^{n} \times \mathbf{S}(n)$.

If not, we substitute $u(t, x)=w(t, x) e^{B t}$ (with $B=(\lambda+\gamma) / \beta$ ) into the equation in (1.1), then we have

$$
g\left(t, x ; w, w_{t}(\tau) ; \partial_{t} w, D w, D^{2} w\right)=0, \quad t>0, x \in \Omega,
$$

while

$$
g=e^{-B t} f\left(t, x ; e^{B t} w,\left(e^{B t} w\right)_{t}(\tau) ; L e^{B t} w+e^{B t} \partial_{t} w, e^{B t} D w, e^{B t} D^{2} w\right)
$$

which satisfies (4.4), where

$$
\left(e^{B t} w\right)_{t}(\tau)=\left(e^{B\left(t+\tau_{1}\right)} w\left(t+\tau_{1}, x\right), \ldots, e^{B\left(t+\tau_{m}\right)} w\left(t+\tau_{m}, x\right)\right) .
$$

Moreover, such $g\left(t, x ; w, w_{t}(\tau) ; \partial_{t} w, D w, D^{2} w\right)$ satisfies $(\mathrm{H} 2)$ (with a different function $\omega_{1}\left(\alpha|x-y|^{2}+|x-y|\right)$ from $\omega$ for $f$ ) and (H1), (H4) (with the same parameters $\beta$ and $L_{j}, j=1,2, \ldots, m$, for $f$ ). 
Now, define the function set

$$
\mathscr{B}=\left\{\eta \in C\left(\bar{Q}_{\bar{\tau}} \cup \bar{Q}\right) \mid U^{*}(t, x) \leq \eta(t, x) \leq V_{*}(t, x),(t, x) \in \bar{Q}_{\bar{\tau}} \cup \bar{Q}\right\} .
$$

It can be seen that $\mathscr{B}$ is a bounded subset of $C\left(\bar{Q}_{\bar{\tau}} \cup \bar{Q}\right)$ because $U^{*}$ and $V_{*}$ are bounded on a compact domain $\bar{Q}_{\bar{\tau}} \cup \bar{Q}$, and the operator $A: \mathscr{B} \rightarrow \mathscr{B}$.

To prove that $A$ is a contraction mapping, let $u=A \eta, v=A \xi$, for $\eta, \xi \in \mathscr{B}$.

We assume that

$$
\sup _{(t, x) \in Q_{\bar{\tau}} \cup Q}\{u(t, x)-v(t, x)\}>0,
$$

and consider the function

$$
M(t, x, y)=u(t, x)-v(t, y)-\frac{\alpha}{2}|x-y|^{2} .
$$

Suppose that $\left(t_{\alpha}, x_{\alpha}, y_{\alpha}\right)$ is the maximum point of $M(t, x, y)$ in $[-\bar{\tau}, T] \times \bar{\Omega} \times \bar{\Omega}$ for a fixed $\alpha$. This maximum, denoted by $M_{\alpha}$, can be obtained and is bounded since $M(t, x, y)$ is upper semicontinuous and the domain $[-\bar{\tau}, T] \times \bar{\Omega} \times \bar{\Omega}$ is compact.

From the initial-boundary value conditions, we conclude that $t_{\alpha} \notin[-\bar{\tau}, 0]$ and $x_{\alpha}, y_{\alpha} \notin$ $\partial \Omega$ if $\alpha$ is large enough. Thus, we can apply [1, Theorem 8.3] to see that there are $a \in \mathbb{R}$, $p=q=\alpha\left(x_{\alpha}-y_{\alpha}\right) \in \mathbb{R}^{n}, X, Y \in \mathbf{S}(n)$ such that

$$
(a, p, X) \in \overline{\mathscr{P}}_{\Omega}^{2,+} U^{*}\left(t_{\alpha}, x_{\alpha}\right), \quad(a, q, Y) \in \overline{\mathscr{P}}_{\Omega}^{2,-} V_{*}\left(t_{\alpha}, y_{\alpha}\right) .
$$

Then, from (4.4), we have that

$$
\begin{aligned}
& \lambda\left(u\left(t_{\alpha}, x_{\alpha}\right)-v\left(t_{\alpha}, y_{\alpha}\right)\right) \\
& \leq f_{*}\left(t_{\alpha}, x_{\alpha} ; u\left(t_{\alpha}, x_{\alpha}\right), \eta_{t_{\alpha}}\left(\tau, x_{\alpha}\right) ; a, p, X\right) \\
&-f^{*}\left(t_{\alpha}, x_{\alpha} ; v\left(t_{\alpha}, y_{\alpha}\right), \eta_{t_{\alpha}}\left(\tau, x_{\alpha}\right) ; a, p, X\right) \\
&= f_{*}\left(t_{\alpha}, x_{\alpha} ; u\left(t_{\alpha}, x_{\alpha}\right), \eta_{t_{\alpha}}\left(\tau, x_{\alpha}\right) ; a, p, X\right) \\
&-f^{*}\left(t_{\alpha}, y_{\alpha} ; v\left(t_{\alpha}, y_{\alpha}\right), \xi_{t_{\alpha}}\left(\tau, y_{\alpha}\right) ; a, q, Y\right) \\
&+f^{*}\left(t_{\alpha}, y_{\alpha} ; v\left(t_{\alpha}, y_{\alpha}\right), \xi_{t_{\alpha}}\left(\tau, y_{\alpha}\right) ; a, q, Y\right) \\
&-f_{*}\left(t_{\alpha}, y_{\alpha} ; v\left(t_{\alpha}, y_{\alpha}\right), \eta_{t_{\alpha}}\left(\tau, x_{\alpha}\right) ; a, q, Y\right) \\
&+f_{*}\left(t_{\alpha}, y_{\alpha} ; v\left(t_{\alpha}, y_{\alpha}\right), \eta_{t_{\alpha}}\left(\tau, x_{\alpha}\right) ; a, q, Y\right) \\
&-f^{*}\left(t_{\alpha}, x_{\alpha} ; v\left(t_{\alpha}, y_{\alpha}\right), \eta_{t_{\alpha}}\left(\tau, x_{\alpha}\right) ; a, p, X\right) .
\end{aligned}
$$

Using (2.9) and (H2), (H4) to estimate three terms on the right, respectively, we have that $(4.12)<0$ and

$$
\begin{gathered}
(4.14) \leq \omega\left(\alpha\left|x_{\alpha}-y_{\alpha}\right|^{2}+\left|x_{\alpha}-y_{\alpha}\right|\right) \\
(4.13) \leq \sum_{j=1}^{m} L_{j}\left|\xi\left(t_{\alpha}+\tau_{j}, y_{\alpha}\right)-\eta\left(t_{\alpha}+\tau_{j}, x_{\alpha}\right)\right|
\end{gathered}
$$


3546 Viscosity solutions to functional parabolic PDE

By [1, Lemma 3.1], there is a point $(\hat{t}, \hat{x})$ such that both $\left(t_{\alpha}, x_{\alpha}\right)$ and $\left(t_{\alpha}, y_{\alpha}\right)$ go to $(\hat{t}, \hat{x})$ as $\alpha \rightarrow \infty$, and

$$
\begin{gathered}
u(\hat{t}, \hat{x})-v(\hat{t}, \hat{x})=\sup _{(t, x) \in Q_{\bar{\tau}} \cup Q}(u(t, x)-v(t, x)), \\
\lim _{\alpha \rightarrow+\infty} \alpha\left|x_{a}-y_{a}\right|=0, \\
\lim _{\alpha \rightarrow+\infty}\left(u\left(t_{\alpha}, x_{\alpha}\right)-v\left(t_{\alpha}, y_{\alpha}\right)\right)=\sup _{(t, x) \in Q_{\bar{\tau}} \cup Q}(u(t, x)-v(t, x)) .
\end{gathered}
$$

Thanks to $\omega\left(0^{+}\right)=0$, the following holds:

$$
\lambda \sup _{(t, x) \in Q_{\bar{\tau}} \cup Q}(u-v) \leq \sum_{j=1}^{m} L_{j}\left|\xi\left(\hat{t}+\tau_{j}, \hat{x}\right)-\eta\left(\hat{t}+\tau_{j}, \hat{x}\right)\right| \leq L \sup _{(t, x) \in Q_{\bar{\tau}} \cup Q}|\xi-\eta|
$$

where $L=\sum_{j=1}^{m} L_{j}$.

In the same manner, we can show that

$$
\lambda \sup _{(t, x) \in Q_{\bar{\tau}} \cup Q}(v(t, x)-u(t, x)) \leq L \sup _{(t, x) \in Q_{\bar{\tau}} \cup Q}|\xi(t, x)-\eta(t, x)| .
$$

We can assume that $\lambda>L$ in (4.4). Then, there is a positive number $\delta=L / \lambda<1$ such that

$$
\|u-v\|<\delta\|\xi-\eta\|
$$

Hence, $A$ is a contraction mapping. By Banach's fixed point theorem, there is a unique fixed point for $A$ in $C\left(\bar{Q}_{\bar{\tau}} \cup \bar{Q}\right)$, which is a unique viscosity solution of (1.1).

Because (1.1) has a viscosity solution on $\left[-\bar{\tau}, T_{1}\right) \times \Omega$ with any finite $T_{1}$, we consider the following problem:

$$
\begin{gathered}
f\left(t, x ; u, u_{t}(\tau) ; \partial_{t} u, D_{x} u, D_{x}^{2} u\right)=0, \quad(t, x) \in\left(T_{1},+\infty\right) \times \Omega, \\
u(t, x)=0, \quad(t, x) \in\left(T_{1},+\infty\right) \times \partial \Omega, \\
u(t, x)=\phi(t, x), \quad(t, x) \in\left[-\bar{\tau}, T_{1}\right] \times \Omega .
\end{gathered}
$$

From the above result, the solution can be extended through to the domain $\left[-\bar{\tau}, 2 T_{1}\right) \times \Omega$. In such a way, the solution can be extended over $[-\bar{\tau},+\infty) \times \Omega$.

The uniqueness follows from the comparison. Thus, the theorem is proved. 
Remark 4.2. From the proof above, it can be seen easily that some of conditions (H1)(H4) could be weakened furthermore, for example, (H1) could be replaced by the following condition.

(H1') There exists a real number $\beta>0$ such that

$$
f_{*}(t, x ; r, W ; a, p, X)-f_{*}(t, x ; r, W ; b, p, X) \geq \beta(a-b),
$$

or

$$
f^{*}(t, x ; r, W ; a, p, X)-f^{*}(t, x ; r, W ; b, p, X) \geq \beta(a-b),
$$

for $a \geq b,(t, x ; r, W ; p, X) \in \bar{Q} \times \mathbb{R} \times \mathbb{R}^{m} \times \mathbb{R}^{n} \times \mathbf{S}(n)$, while (H2)-(H4) could be weakened similarly.

\section{Construction of coupled viscosity sub- and super-solutions in the strong sense}

In this section, we are going to construct coupled viscosity sub- and super-solutions in the strong sense provided there are continuous viscosity sub- and super-solutions.

Suppose here that $\Omega$ is a bounded convex domain, and there are continuous viscosity sub- and super-solutions $U(t, x)$ and $V(t, x)$ of $(1.1)$ with $U(t, x)=V(t, x)=0$ on $\partial \Omega$ and an additional assumption such as $V(t, x)-U(t, x) \leq C D(x)$ for all $x$ near $\partial \Omega$ for $\tau<t<T$, where $D(x)$ is the distance of a point from the boundary and $C$ is a suitable constant. Thus, $V(t, x)-U(t, x)$ is uniform continuous on $\bar{Q}_{\tau}$ and $d=\sup _{Q_{\bar{\tau}}}|V(t, x)-U(t, x)|<$ $+\infty$.

From the theory of PDE, it can be seen that there is an eigenfunction $\phi(x)$ corresponding to the first eigenvalue of the following eigenvalue problem:

$$
\begin{gathered}
-\triangle \phi=\lambda \phi, \quad x \in \Omega, \\
\phi(x)=0, \quad x \in \partial \Omega,
\end{gathered}
$$

which satisfies that $\phi(x)>V(t, x)-U(t, x) \geq 0$ on $x \in \Omega_{\delta}$ for $t>\tau$, where

$$
\Omega_{\delta}=\{x \in \Omega|| x-y \mid<\delta, y \in \partial \Omega\}
$$

and $-D^{2} \phi(x) \geq 0$.

Thus, from the compactness of $Q$ and the continuity of $V-U$, it can be seen that

$$
M=\sup _{Q_{\bar{\tau}}} \frac{V(t, x)-U(t, x)}{\phi(x)}<+\infty .
$$

Assume that $h(t) \in C^{1}[-\bar{\tau}, T)$ is a positive solution of the following retarded ordinary differential equation:

$$
\beta h^{\prime}-\lambda h-\sum_{j=1}^{m} L_{j}\left(h\left(t+\tau_{j}\right)+M\right) \geq 0 .
$$


3548 Viscosity solutions to functional parabolic PDE

There is a positive solution to (5.4). Taking notice of the fact that $\tau_{j}<0, j=1, \ldots, m$, we see that

$$
\lim _{\lambda \rightarrow+\infty} \sum_{j=1}^{m} L_{j} e^{\lambda \tau_{j}}=0
$$

Hence, there is a number $L$ large enough such that

$$
\left(\beta L-\gamma-\sum_{j=1}^{m} L_{j}\left(e^{L \tau_{j}}+M\right)\right) e^{L t} \geq d \sum_{j=1}^{m} L_{j}
$$

That is, $h(t)=e^{L t}$ is positive solution to the inequality (5.4).

Let

$$
\begin{aligned}
\hat{U}(t, x)=U(t, x)-h(t) \phi(x), & \hat{V}(t, x)=V(t, x)+h(t) \phi(x) \\
\hat{a}=a-h^{\prime}(t) \phi(x), & \hat{b}=b+h^{\prime}(t) \phi(x), \\
\hat{p}=p-h(t) D \phi, & \hat{q}=q+h(t) D \phi, \\
\hat{X}=X-h(t) D^{2} \phi, & \hat{Y}=Y+h(t) D^{2} \phi .
\end{aligned}
$$

We observe that

$$
\begin{aligned}
& \mathscr{P}_{\Omega}^{2,+} \hat{U}(t, x)=\left\{(\hat{a}, \hat{p}, \hat{X}) \mid(a, p, X) \in \mathscr{P}_{\Omega}^{2,+} U(t, x)\right\}, \\
& \mathscr{P}_{\Omega}^{2,-} \hat{V}(t, x)=\left\{(\hat{b}, \hat{q}, \hat{Y}) \mid(b, q, Y) \in \mathscr{P}_{\Omega}^{2,-} V(t, x)\right\} .
\end{aligned}
$$

For any continuous function $\eta$ between $\hat{U}$ and $\hat{V}$, we observe that

$$
\begin{aligned}
& |\eta(t, x)-U(t, x)| \leq h(t) \phi(x)+V(t, x)-U(t, x), \\
& |\eta(t, x)-V(t, x)| \leq h(t) \phi(x)+V(t, x)-U(t, x),
\end{aligned} \quad \text { on } \bar{Q}_{\bar{\tau}} .
$$

We assume that $f$ satisfies (H1)-(H4) and is independent of $D u$. Then,

$$
\begin{aligned}
f_{*}(t, x ; & \left.U, U_{t}(\tau) ; a, X\right)-f_{*}\left(t, x ; \hat{U}, \eta_{t}(\tau) ; \hat{a}, \hat{X}\right) \\
= & f_{*}\left(t, x ; U, U_{t}(\tau) ; a, X\right)-f_{*}\left(t, x ; U, U_{t}(\tau) ; a, \hat{X}\right) \\
& +f_{*}\left(t, x ; U, U_{t}(\tau) ; a, \hat{X}\right)-f_{*}\left(t, x ; U, U_{t}(\tau) ; \hat{a}, \hat{X}\right) \\
& +f_{*}\left(t, x ; U, U_{t}(\tau) ; \hat{a}, \hat{X}\right)-f_{*}(t, x ; \hat{U}, U(\tau) ; \hat{a}, \hat{X}) \\
& +f_{*}\left(t, x ; \hat{U}, U_{t}(\tau) ; \hat{a}, \hat{X}\right)-f_{*}\left(t, x ; \hat{U}, \eta_{t}(\tau) ; \hat{a}, \hat{X}\right) .
\end{aligned}
$$

Because $f$ is parabolic and $X \leq \hat{X}$, we see that

$$
(5.10) \geq 0 \text {. }
$$


Then, by (H1), (H3), and (H4),

$$
\begin{aligned}
f_{*}\left(t, x ; U, U_{t}(\tau) ; a, X\right)-f_{*}\left(t, x ; \hat{U}, \eta_{t}(\tau) ; \hat{a}, \hat{X}\right) \\
\geq \phi(x)\left(\beta h^{\prime}(t)-\gamma h(t)-\sum_{j=1}^{m} L_{j}\left(h\left(t+\tau_{j}\right)+M\right)\right) \geq 0, \\
\forall(t, x) \in(0,+\infty) \times \Omega, \quad(a, p, X) \in \mathscr{P}_{\Omega}^{2,+} U(t, x) .
\end{aligned}
$$

Similarly,

$$
\begin{aligned}
f^{*}\left(t, x ; \hat{V}, \eta_{t}(\tau) ; \hat{b}, \hat{Y}\right)-f^{*}\left(t, x ; V, V_{t}(\tau) ; b, Y\right) \\
\geq \phi(x)\left(\beta h^{\prime}(t)-\gamma h(t)-\sum_{j=1}^{m} L_{j}\left(h\left(t+\tau_{j}\right)+M\right)\right) \geq 0, \\
\quad \forall(t, x) \in(0,+\infty) \times \Omega, \quad(b, q, Y) \in \mathscr{P}_{\Omega}^{2,-} V(t, x) .
\end{aligned}
$$

Due to the fact that $U$ and $V$ are viscosity sub- and super-solutions to (1.1), respectively, we have that

$$
\begin{gathered}
f_{*}\left(t, x ; \hat{U}, \eta_{t}(\tau) ; \hat{a}, \hat{X}\right) \leq 0, \\
f^{*}\left(t, x ; \hat{V}, \eta_{t}(\tau) ; \hat{b}, \hat{Y}\right) \geq 0, \\
\forall(t, x) \in(0, T) \times \Omega, \\
(\hat{a}, \hat{p}, \hat{X}) \in \mathscr{P}_{\Omega}^{2,+} \hat{U}(t, x), \quad(\hat{b}, \hat{q}, \hat{Y}) \in \mathscr{P}_{\Omega}^{2,-} \hat{V}(t, x) .
\end{gathered}
$$

Obviously, $\hat{U}=\hat{V}=0$ on $\partial \Omega$ for $t>\bar{\tau}$. Thus, we have the following assertion.

Theorem 5.1. Suppose that $\Omega$ is a bounded convex domain, $f$ is as mentioned above and independent of Du. Let $U$ and $V$ be continuous viscosity sub-and super-solutions of (1.1), respectively. Then, there is coupled viscosity sub-and super-solutions in the strong sense to (1.1).

\section{Applications}

Now, consider the following initial-boundary value problem for the retarded Bellman equations:

$$
\begin{gathered}
\partial_{t} u+\sup _{\alpha \in \mathscr{A}}\left\{\mathscr{L}^{\alpha} u\right\}=0, \quad(t, x) \in Q_{T}, \\
\left.u\right|_{\partial \Omega}=0, \quad(t, x) \in \Gamma \\
u(t, x)=\phi(t, x), \quad(t, x) \in Q_{\bar{\tau}}
\end{gathered}
$$

where $\mathscr{A}$ is an index set and

$$
\mathscr{L}^{\alpha} u=-\sum_{i, j=1}^{n} a_{i, j}^{\alpha} \frac{\partial^{2} u}{\partial x_{i} \partial x_{j}}+\sum_{k=0}^{m} c_{k}^{\alpha} u_{t}\left(\tau_{k}\right)-f^{\alpha},
$$

which depends on the retarded term $\sum_{k=0}^{m} c_{k}^{\alpha} u_{t}\left(\tau_{k}\right)$. 
Suppose that

(i) $\Omega$ is a bounded convex domain in $\mathbb{R}^{n}$;

(ii) all coefficients $\left\{a_{i, j}^{\alpha}\right\}_{\alpha},\left\{b_{i}^{\alpha}\right\}_{\alpha},\left\{c_{k}^{\alpha}\right\}_{\alpha},\left\{f^{\alpha}\right\}_{\alpha}$ are $n^{2}+n+m+1$ equicontinuous function sets in $\bar{Q}$;

(iii) $\sum_{i, j=1}^{n} a_{i, j}^{\alpha} \xi_{i} \xi_{j} \geq 0$, that is, the matrices $\left(a_{i j}^{\alpha}\right)$ are positive semidefinite.

The equation is degenerate parabolic and the conditions $(\mathrm{H} 1)-(\mathrm{H} 4)$ hold thanks to the supposition. We observe that there is a number $M$ large enough such that $U(t, x)=$ $-e^{M t} \phi(x)$ and $V=e^{M t} \phi(x)$ are sub- and super-solutions to (6.1), respectively, where $\phi(x)$ is an eigenfunction corresponding to the first eigenvalue of the eigenvalue problem (5.1).

Then, from Theorem 4.1, we know that there is a unique viscosity solution of (6.1).

For the Iscaas equations, we have the similar conclusion.

\section{Acknowledgments}

Authors would like to thank the referee for comment and helpful revision suggestion. This work was supported by the National Natural Science Foundation of China, no. 10471108 .

\section{References}

[1] M. G. Crandall, H. Ishii, and P.-L. Lions, User's guide to viscosity solutions of second order partial differential equations, Bull. Amer. Math. Soc. (N.S.) 27 (1992), no. 1, 1-67.

[2] H. Engler and S. M. Lenhart, Viscosity solutions for weakly coupled systems of Hamilton-Jacobi equations, Proc. London Math. Soc. (3) 63 (1991), no. 1, 212-240.

[3] H. Ishii and S. Koike, Viscosity solutions for monotone systems of second-order elliptic PDEs, Comm. Partial Differential Equations 16 (1991), no. 6-7, 1095-1128.

[4] L. Jiang and M. Dai, Convergence of binomial tree method for American options, Partial Differential Equations and Their Applications (Wuhan, 1999) (H. Chen and L. Rodino, eds.), World Scientific, New Jersey, 1999, pp. 106-118.

[5] G. Lu and B. D. Sleeman, Maximum principles and comparison theorems for semilinear parabolic systems and their applications, Proc. Roy. Soc. Edinburgh Sect. A 123 (1993), no. 5, 857885.

[6] Subsolutions and supersolutions to systems of parabolic equations with applications to generalized Fujita-type systems, Math. Methods Appl. Sci. 17 (1994), no. 13, 1005-1016.

[7] Y. Qixiao and L. Zhengyuan, Introduction of Reaction-Diffusion Equations, Sinica Sci. Press, Beijing, 1994.

Liu Wei-an: School of Mathematics and Statistics, Wuhan University, Wuhan, Hubei 430072, China E-mail address: liuweian@public.wh.hb.cn

Lu Gang: School of Mathematics and Statistics, HuaZhong Normal University, Wuhan, Hubei 430079, China

E-mail address: glu@e21.edu.cn 


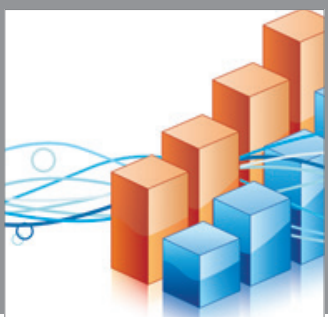

Advances in

Operations Research

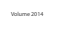

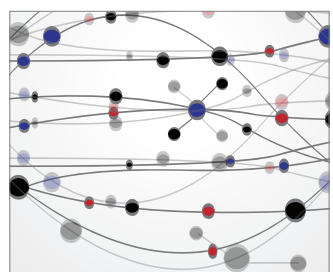

\section{The Scientific} World Journal
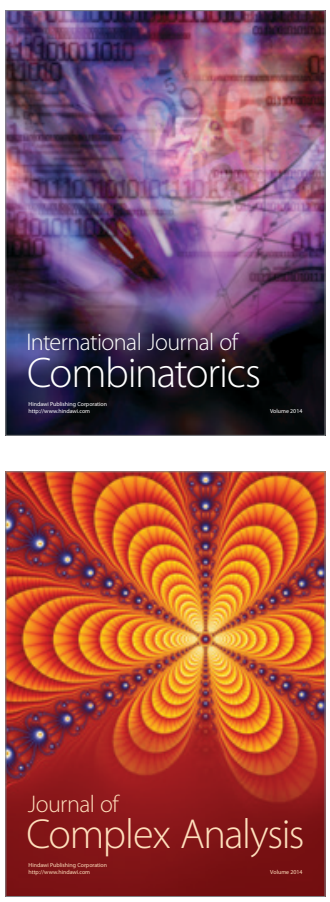

International Journal of

Mathematics and

Mathematical

Sciences
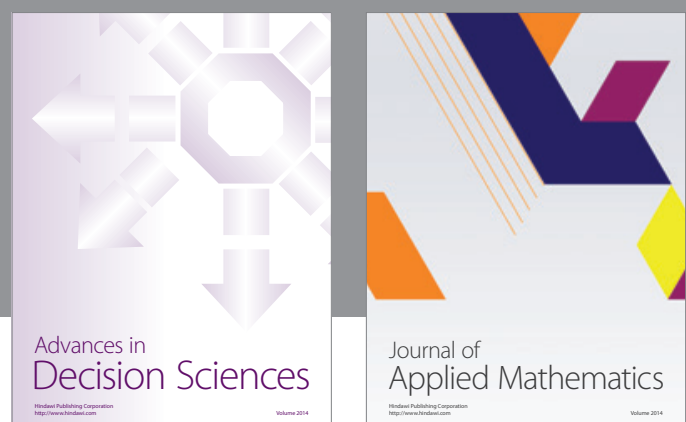

Journal of

Applied Mathematics
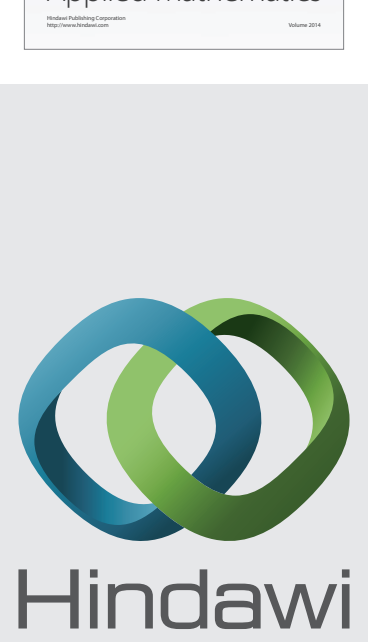

Submit your manuscripts at http://www.hindawi.com
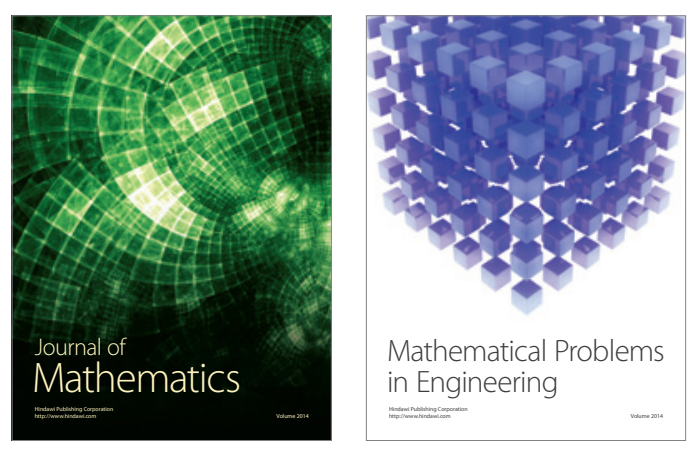

Mathematical Problems in Engineering
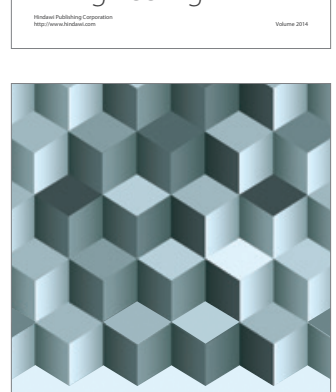

Journal of

Function Spaces
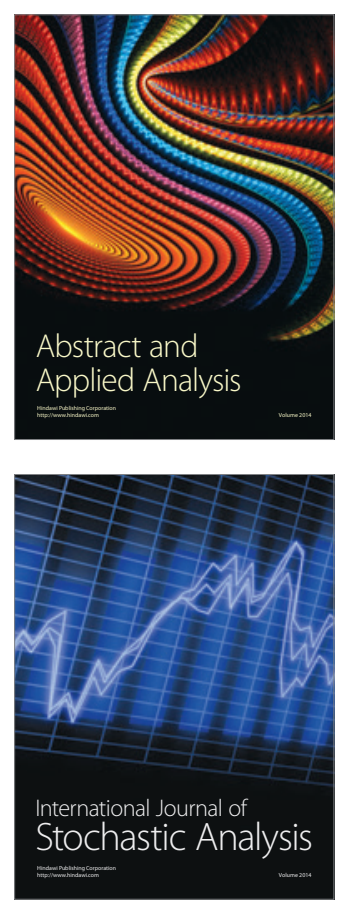

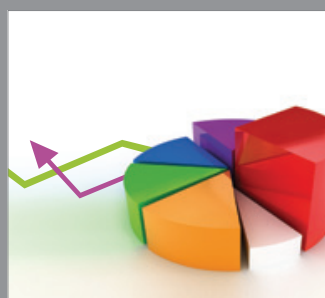

ournal of

Probability and Statistics

Promensencen
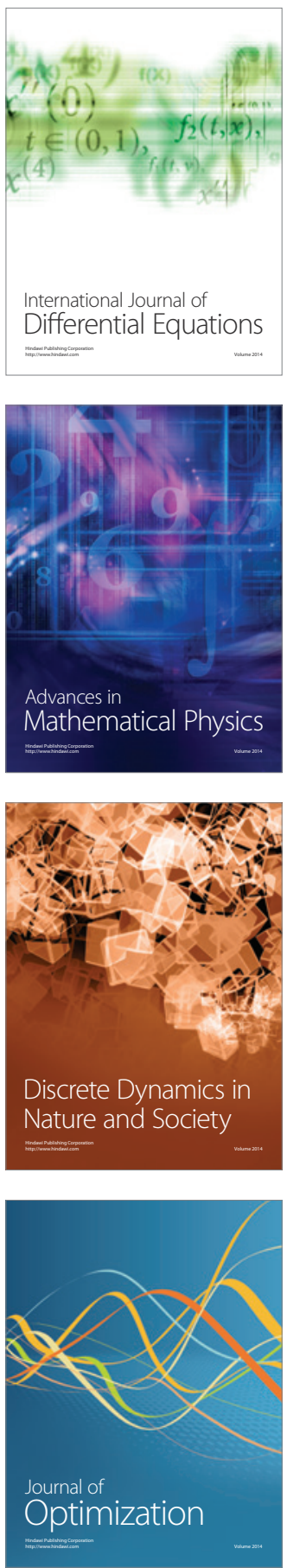